DOI: $10.17957 / \mathrm{IJAB} / 15.1798$

http://www.fspublishers.org

\title{
Identification of Drought-Responsive CircRNAs in Leaves of Brachypodium distachyon
}

\author{
Yalan Feng ${ }^{1,2 \dagger}$, Shuang Zhou ${ }^{1,2}$, Jun Zhang ${ }^{1,2}, \mathrm{Ke} \mathrm{Xv}^{3}$, Yating $\mathrm{Li}^{1,2}$, Mengzhen Zhang ${ }^{1,2}$, Youjun $\mathrm{Li}^{1,2}$ and $\mathrm{Chao} \mathrm{Ma}^{1,2^{*}}$ \\ ${ }^{1}$ College of Agriculture, Henan University of Science and Technology, Luoyang 471023, Henan province, P. R. China \\ ${ }^{2}$ Dry-Land Agricultural Engineering Technology Research Center in Henan, Luoyang 471023, Henan province, P. R. China \\ ${ }^{3}$ Department of Agriculture and Rural Affairs of Shanzhou District, Sanmenxia 472100, Henan province, P. R. China \\ "For correspondence: machao840508@163.com \\ Contributed equally to this work and are co-first authors \\ Received 21 January 2020; Accepted 24 March 2021; Published 10 May 2021
}

\begin{abstract}
Brachypodium distachyon is currently recognized as a model organism in Gramineae, especially for temperate grains. Research on its dehydration response mechanism is beneficial for elucidating the complex drought regulation network in temperate grains. The circRNA is an important type of non-coding RNA in organisms, which could involve in various biological processes. By sequencing the transcriptome of the leaves of $B$. distachyon under desiccation treatment and normal condition, a total of 737 circRNAs were obtained in this study, of which 332 and 265 were up-regulated and 265 downregulated, respectively, with 140 insignificant differences. There were 229 and 303 circRNAs specifically expressed under CK and drought, respectively and 204 were co-expressed. Bioinformatics methods were used to identify potential miRNA targets of differentially expressed circRNAs. Among them, 150 differentially expressed circRNAs had putative miRNA binding sites. According to the predicted miRNA with binding site, the target genes downstream of the miRNA were screened. In terms of the functional annotation of target genes, they are divided into seven categories: hormone-related, photosystem, stress response, regulation factor, ion transport, TF and ribosomal protein. These circRNAs involved in water deficit response may play a significant role in the drought response and defense of $B$. distachyon. This also provides new insights for the improvement of the dehydration regulation mechanism of $B$. distachyon and even temperate grains. (C) 2021 Friends Science Publishers
\end{abstract}

Keywords: Brachypodium distachyon; circRNAs; Drought; Illumina sequencing; Functional category

\section{Introduction}

Compared with the well-known model plants Arabidopsis thaliana and rice, Brachypodium distachyon (B. distachyon) is more closely related to many important cereal crops such as wheat, barley, oats, corn, and so on (Brkljacic et al. 2011; Catalan et al. 2015). Therefore B. distachyon is a currently recognized model organism for Gramineae, and its biological characteristics, such as easy cultivation, smaller habit, and short growth cycle (Scholthof et al. 2018), are conducive to in-depth research on the development, evolutionary biology and abiotic stress response of temperate grain crops.

Unlike linear RNA, circular RNA (circRNA) is a special member of the non-coding RNA family. Since circRNA forms a covalent closed loop structure and does not have a polyadenylic acid tail, it is resistant to RNase, which can effectively degrade linear RNA (Zhao et al. 2019). The discovery of circRNA has been for decades, and it was once considered a by-product of splicing errors (Sanger et al. 1976; Kos et al. 1986). However, with the continuous development of high-throughput deep sequencing technology and bioinformatics tools, a variety of circRNAs have been discovered and identified in many organisms, such as humans (Jeck et al. 2013), fruit flies (Westholm et al. 2014), mice (Fan et al. 2015), zebrafish (Shen et al. 2017), Arabidopsis (Ye et al. 2015), rice (Lu et al. 2015) and soybeans (Wang et al. 2020). These researches confirmed that circRNA is ubiquitous in eukaryotes. The recent research on circRNA is mostly concentrated in mammals (Memczak et al. 2013), whereas research in plants is still in its infancy. The whole genome identification of circRNA in plants was first carried out in Arabidopsis thaliana and Oryza sativa. Ye et al. $(2015,2016)$ identified 6,012 and 2,806 circRNAs from Arabidopsis leaves and rice seedling root tissues, respectively. In addition to these two well-known model plants, circRNA has also been identified in other monocot and dicot species, such as soybean (Wang et al. 2020), barley (Behrooz et al. 2016), and wheat (Xu et al. 2019). Similar to the expression pattern of circRNA in animals (Westholm et al. 2014; Fan et al. 2015), circRNA in plants also exhibits tissue specificity and responds to environmental stress (Lu et al. 2015; Behrooz et al. 2016). 
For example, rice has 27 different expressions of circRNA under conditions of sufficient phosphate and starvation (Ye et al. 2015). There are 163 circRNAs in tomato showing a cold-responsive expression pattern (Zuo et al. 2016). Increasing evidences indicate that circRNAs may play significant roles in a variety of biological processes, such as miRNA binding, protein binding and transcriptional regulation (Hansen et al. 2013; Memczak et al. 2013; Chen et al. 2016).

Drought is one of the most serious adversity stresses affecting plant growth. With the global warming, the shortage of water resources has become more and more serious, which has directly led to the expansion of arid areas and the increase of aridity. In the long-term evolutionary process, plants have formed their own defense response mechanisms to deal with various stresses including water privation, such as the accumulation of osmotic adjustment compounds (Sattar et al. 2020b), stoma regulation systems (Sattar et al. 2020a), and signal transduction pathways (Iqbal et al. 2017). However, the response of plants to drought stress is the result of multiangle and multi-level interaction, including not only the transcription level and translation level, but also the regulation of the post-transcriptional level (Chen et al. 2017b). As a result, in addition to mRNA, there are also a large number of non-coding RNAs involved, such as miRNA, IncRNA and circRNA. The researches about the former two in drought stress have been widely reported (Ma et al. 2019; Nadarajah and Kumar 2019; Feng et al. 2020), whereas research on how circRNA functions in drought stress response and regulation is rare. Therefore, this study adopted high-throughput technology to sequence the transcriptome of the $B$. distachyon leaves under water shortage stress, and used bioinformatics tools to analyze and identify the sequencing results, intending to provide theoretical basis for further understanding and improvement of drought response in Gramineous crops.

\section{Materials and Methods}

\section{Plant material, growing environment and drought treatment}

B. distachyon $\mathrm{Bd}-21$ was sprouted in a clean petri dish for 1 day and then transferred to a $4^{\circ} \mathrm{C}$ incubator for 8 days for vernalization. After vernalization, these seedlings were planted in a pot, placed in a light incubator at $25^{\circ} \mathrm{C}$ with photoperiod $16 \mathrm{~h}$ light/ $8 \mathrm{~h}$ dark. When the seedlings grew to the 7-leaf stage, they were subjected to drought treatment. The control samples were watered once every other day, and the experimental group was treated with water restriction in a drought treatment for 7 days. After the treatment, the leaves of the seedlings were quickly frozen in liquid nitrogen and stored in an ultra-low temperature refrigerator at $-80^{\circ} \mathrm{C}$ for subsequent experiment.

\section{Library construction, sequencing and circRNA identification}

RNA sequencing is a transcriptomics research method based on next-generation sequencing technology. Each step of sample detection, library construction, and sequencing is strictly controlled to ensure the output of high-quality data. First, extract the total RNA of the biological sample, according to the Feng et al. (2020) description. Then remove the rRNA and linear RNA. The RNA obtained is theoretically only circular RNA, and then through the steps of reverse transcription and PCR, and finally the enriched cDNA is subjected to high-throughput sequencing. After base calling analysis, the original image data files obtained by Illumina HiSeqTM2500 sequencing platforms are converted into sequenced reads, namely raw reads. CircRNA from the sequencing results could be identified through find circ (Memczak et al. 2013), and the candidate circRNAs whose read count greater than or equal to 2 were taken as the identified circRNA.

\section{Expression analysis of circRNAs}

The expression level of known and new circRNA in each sample was counted, which was normalized by transcript per million tags (TPM) (Zhou et al. 2010). Differentially expressed genes sequencing (DEGseq) (Wang et al. 2010) was employed to analyze the differences in circRNA expression between different samples. The differential circRNAs were screened from two aspects of fold change and corrected significance level (q value). The default differential circRNA screening condition is: $\mathrm{q}$ value $<0.01$ and $\mid \log 2$ (fold change) $\mid>1$.

\section{Verification of circRNA}

Since the splice junction mapping on the genome after reverse splice of circRNA is reversed, the specific primers for detecting circRNA are generally designed as divergent primers, which are correspond to the convergent primers used to detect linear genes. Divergent primers include two types. Type I is generally designed back-to-back so that the splice junction is included in the middle of the product (Fig. 1A). Type II is the junction overlapping divergent primers (JOD primers), which span the splice junction, that is, the splice junction needs to be placed at the 3 'end of a primer so that the splice junction is included on the primer rather than included in the PCR product (Fig. 1B). During PCR, JOD primers can only be amplified normally when they match the target circRNA, and it cannot be amplified when there is a mismatch, which greatly improves the specificity. Convergent primers were also designed as positive controls for linear transcripts (Fig. 1C). The designed primers were amplified and verified using cDNA and gDNA as templates. In order to confirm the reliability of the transcriptome sequencing results, 8 circRNAs were randomly selected for 
qRT-PCR to verify their expression patterns. The highly specific JOD primers were used as primers, and the fluorescence quantification system and procedures were described by Feng et al. (2019) and Ma et al. (2019).

\section{Bioinformatics analysis of parent genes of circRNAs under drought treatment}

The input data of circRNA differential expression was the read count obtained in the analysis of circRNA expression level. The software psRobot and psRNATarget were adopted to predict the miRNA binding site of circRNA (Hansen et al. 2013) and the target gene of miRNA (Dai and Zhao 2011), respectively. After the differentially expressed circRNAs between each group were acquired, according to the corresponding relationship between circRNA and predicted miRNAs with circRNA binding sites, as well as target genes downstream of miRNAs, the Gene Ontology (GO) and Kyoto Encyclopedia of Genes and Genomes (KEGG) enrichment analyses were carried out (Zhang et al. 2017). GO (http://www.geneontology.org/) is an international standard classification system for gene function. KEGG is the major public database about pathway (Kanehisa et al. 2008). Pathway significant enrichment analysis takes KEGG pathway as the unit and applies hypergeometric test to find the pathway that is significantly enriched in the target gene compared with the entire genome background.

\section{Results}

\section{Sequencing results statistics and circRNA identification}

To identify the circRNAs in $B$. distachyon responsing to drought stress, we constructed the RNA-Seq library from drought-treated $B$. distachyon leaves and performed Illumina HiSeq high-throughput sequencing. The above library construction, sequencing and bioinformatics analysis were carried out by Beijing Novogene Bioinformatics Co., Ltd. Each step of sample detection, library construction, and sequencing is strictly controlled to ensure the output of high-quality data. The quality assessment of the original data of the sample sequencing output is shown in Table 1. The CK and clean reads under water loss are 122,429,492 and $111,457,314$, respectively. Clean bases reached $18.36 \mathrm{G}$ and $16.72 \mathrm{G}$, respectively. The length distribution of circRNA of all samples is shown in Fig. 2. It can be seen from the Fig. 2 that the length of circRNA obtained by sequencing is mainly concentrated below 1000nt, and the number gradually decreases as the length increases. According to the source of circRNA in the genome and its constituent sequence, it can be divided into exonic circRNAs, intronic circRNAs and intergenic circRNAs. Fig. 3 counts the sources of circRNA in CK and water scarcity. Among them, There were 870 and 1,111 exonic circRNAs in CK and drought, respectively, which are dominated (53.28 and 52.85\%), and followed by intronic circRNAs with 710 and 920, and intergenic circRNAs are the least with 53 and 71, respectively.

\section{Analysis of differentially expressed circRNAs under drought}

The expression level of known and new circRNA in each sample was calculated, and the expression level was normalized by TPM (Zhou et al. 2010). The differential circRNA was screened through the fold change and the corrected significance level (padj/qvalue). Fig. 4 (volcano map) can infer the overall distribution of different circRNAs. Among them, 332 and 265 were up-regulated and downregulated separately, and 140 were not dramatically different. The Venn diagram of differential expressed circRNAs under CK and drought treatment is shown in Fig. 5. There are 229 and 303 circRNAs specifically expressed under CK and drought, respectively, and 204 are co-expressed.

\section{Validation of circRNA in B. distachyon}

Fluorescence quantitative polymerase chain reaction (qRTPCR) was performed to validate the 8 circRNAs randomly selected from the circRNA-Seq analysis. Using highly specific JOD primers to amplify circRNA, cDNA and gDNA were used as amplification templates, and gDNA was used as negative control (Fig. 6A). Since the JOD primer spans the circularization site, the corresponding fragment cannot be amplified by using gDNA as a template. $\beta$-actin was used as an internal reference gene to normalize the expression of these 8 circRNAs. The qRT-PCR results in Fig. 6B showed that the expression levels of these 8 circRNAs were consistent with the RNA-seq results, indicating the reliability of the RNA-seq results. All the primers were showed in the Supplementary Table 2.

\section{miRNA binding site analysis}

The circRNA can inhibit the function of miRNA by binding to miRNA (Hansen et al. 2013), that is, it can regulate gene expression by acting as a miRNA sponge. Therefore, the miRNA binding site analysis of the identified circRNA will help to further study the function of circRNA. To investigate whether the circRNA in B. distachyon can affect the posttranscriptional level of target genes by binding to miRNAs under dehydration treatment, a bioinformatics method was employed to identify potential miRNAs target position of circRNA that are differentially expressed in B. distachyon.

In this study, 150 differentially expressed circRNAs have putative miRNA binding sites, and each circRNA has at least one predicted miRNA binding site, and a single miRNA can be targeted by multiple circRNAs, and a single circRNA can also target different miRNAs (Supplementary Table 1). For example, the predicted bdi-miR5169a is targeted by 9 circRNAs, while bdi_circ_0000061 can target 3 miRNAs in B. distachyon. In addition, based on predicted 


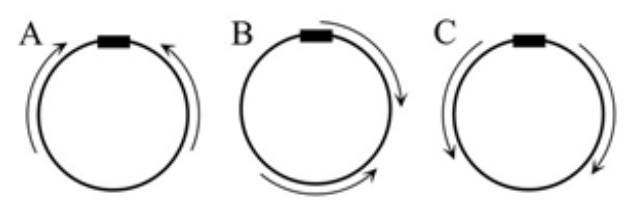

Fig. 1: Schematic diagram of divergent and convergent primers. A, type I divergent primer, the junction site is in the middle of the PCR product; $\mathbf{B}$, type $I$ divergent primer, overlapping with the junction site; $\mathbf{C}$, convergent primer, used to verify linear transcripts. The black rectangle represents the junction site

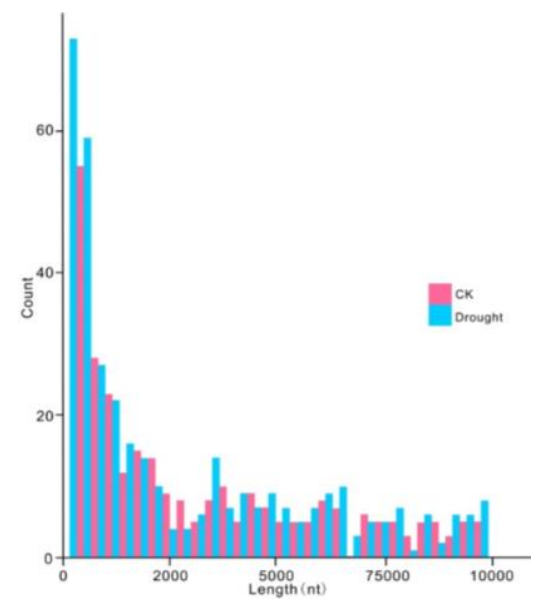

Fig. 2: The length distribution of circRNAs. circRNAs below 1000 nt are 132 and 159 in CK and drought samples, accounting for $40 \%$ and $43 \%$, respectively

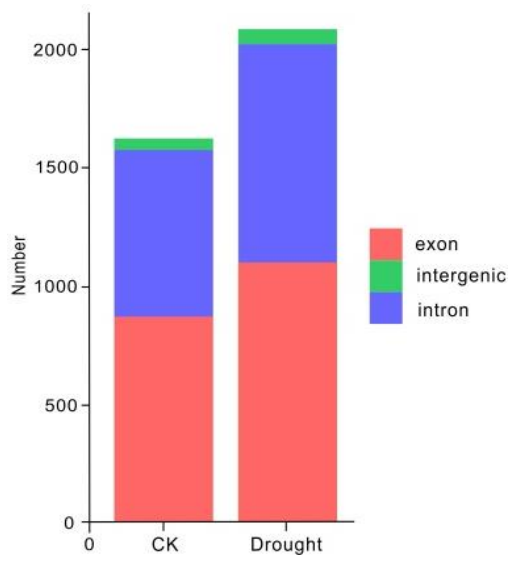

Fig. 3: Statistical analysis of exonic circRNAs, intergenic circRNAs, and intronic circRNAs in each sequenced sample. The above three circRNAs are 870, 53, 710 in CK, and 1111, 71, 920 in drought sample, respectively

miRNAs with binding sites, target genes downstream of miRNAs were screened. In line with function annotations, they are divided into seven categories: hormone-related, photosystem, stress response, regulation factor, ion transport, transcription factor (TF), and ribosomal protein (Table 2, Supplementary Table 2). Among the circRNAs corresponding to these seven types of target genes, the number of up-regulated expression was remarkably more

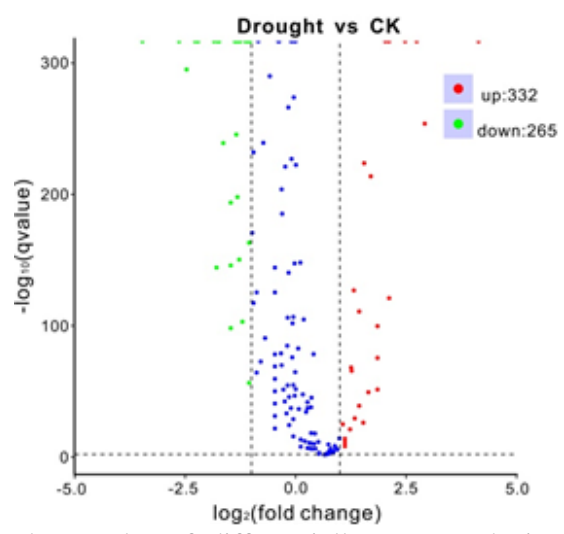

Fig. 4: Volcano plot of differentially expressed circRNA. The abscissa represents the fold change of circRNA expression in different samples, the ordinate represents the statistically significant degree of circRNA expression change, the scattered dots in the figure represent each circRNA, and the blue dots represent circRNAs with no significant differences, the red dots indicate significantly up-regulated circRNAs, and green dots indicate significantly down-regulated circRNAs

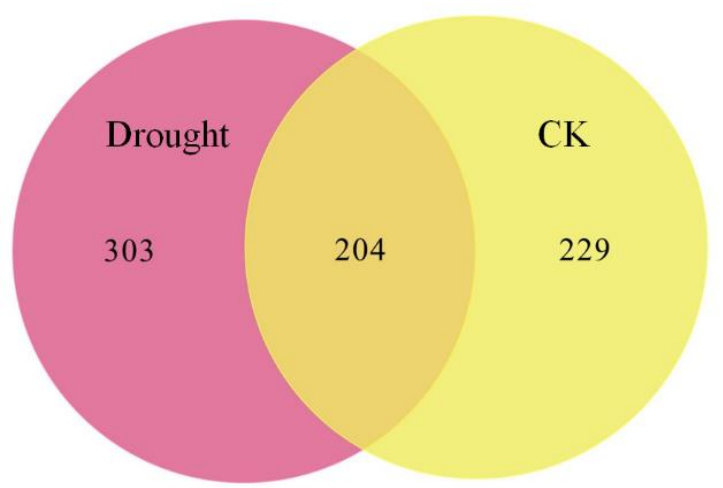

Fig. 5: Venn diagram of differential expressed circRNA. The number in each large circle represents the circRNA expressed in this sample, and the overlapping part of the circle represents the circRNA co-expressed between samples

than that of down-regulated expression (Table 3, Supplementary Table 2).

\section{Bioinformatics analysis of target genes predicted by circRNA under drought}

According to the predicted miRNAs with circRNA binding sites, target genes downstream of miRNAs were screened, and Gene Ontology and KEGG enrichment analysis were performed on these target genes. The number of genes in each GO term that were notably enriched is shown in a histogram (Fig. 7). For biological processes, the category of metabolic process (GO: 0008152) is the richest in GO terms. For cell components, the target genes of circRNA are mainly involved in cell (GO: 0005623) and cell part (GO: 0044464). For molecular functions, the two most abundant categories are binding (GO: 0005488) and catalytic activity (GO: 0003824). The scatter plot of target gene KEGG 

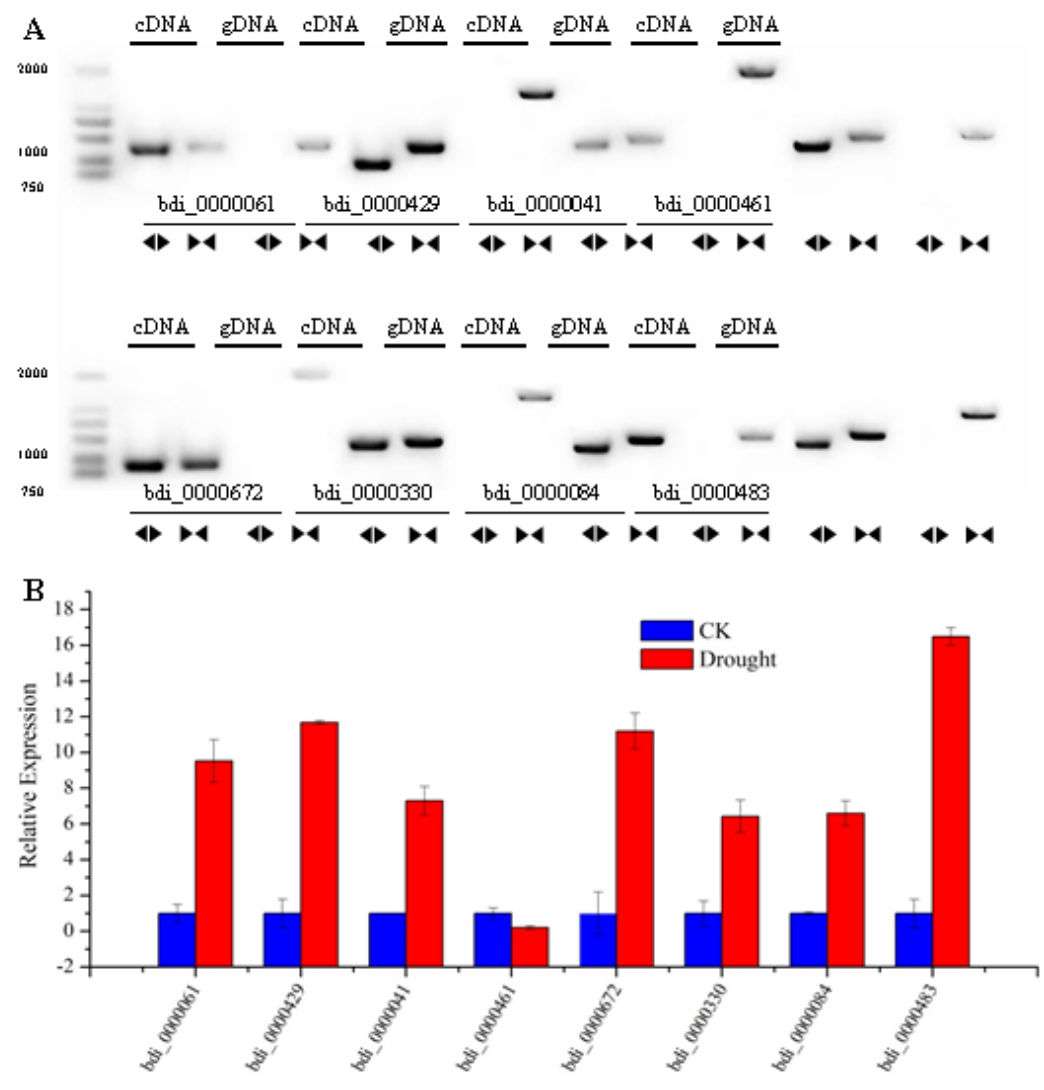

Fig. 6: Various experimental strategies validated the eight circRNAs from transcriptome sequencing. A, JOD primers (black back-to-back triangle pairs) and convergent primers (black opposing triangle pairs) were adopted to amplify eight circRNAs (bdi_0000061, bdi_0000429, bdi_0000041, bdi_0000461, bdi_0000672, bdi_0000330, bdi_0000084, bdi_0000483) in cDNA and gDNA. The former successfully amplified in cDNA but failed in gDNA, and the latter worked on both cDNA and gDNA; B, qRT-PCR validated the expression of the eight circRNAs

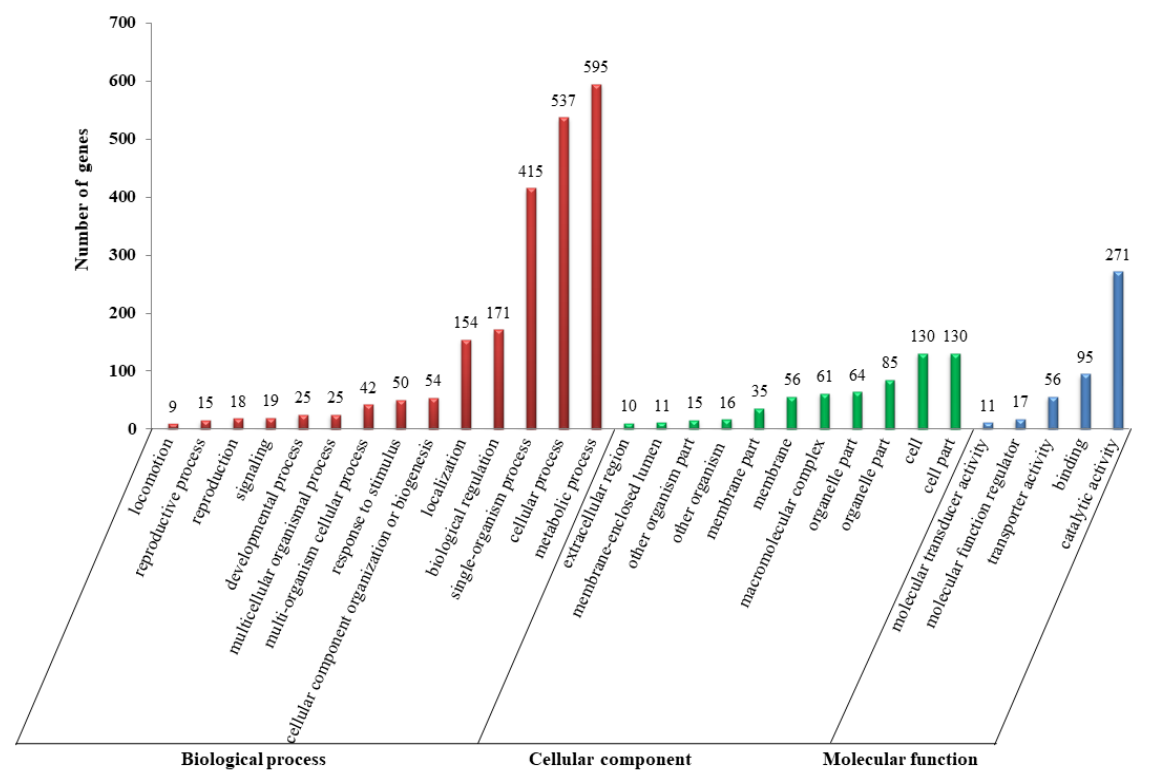

Fig. 7: GO enrichment histogram of target gene predicted by circRNA. The abscissa is the GO term of the three major categories of GO, and the ordinate is the number of target genes annotated to the term (including the sub-terms of the term) 
Table 1: Statistics of sequencing data quality

\begin{tabular}{|c|c|c|c|c|c|c|c|}
\hline Sample & Raw reads & Clean reads & Clean bases & Error rate $(\%)$ & Q20 & Q30 & GC content $(\%)$ \\
\hline CK & $128,474,682$ & $122,429,492$ & $18.36 \mathrm{G}$ & 0.01 & 97.12 & 92.87 & 43.97 \\
\hline Drought & $116,766,272$ & $111,457,314$ & $16.72 \mathrm{G}$ & 0.01 & 97.21 & 92.92 & 44.70 \\
\hline
\end{tabular}

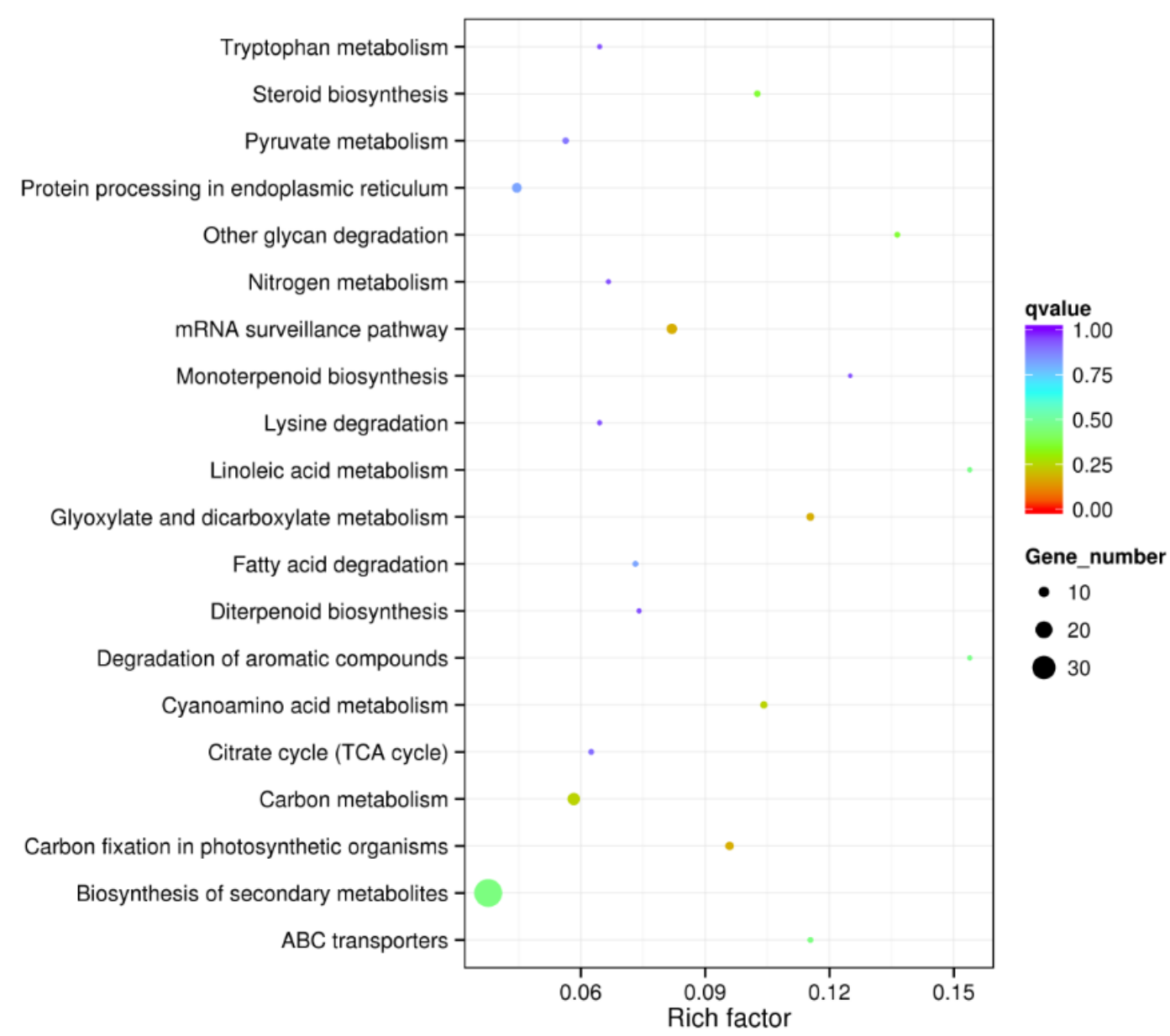

Fig. 8: KEGG enrichment scatter plot of target gene predicted by circRNA. The vertical axis represents the pathway, and the horizontal axis represents the rich factor. The size of the dot indicates the number of target genes in this pathway, and the color of the dot corresponds to different Q-value ranges

enrichment is a graphical display of KEGG enrichment analysis results (Fig. 8). The degree of KEGG enrichment is measured by rich factor, Q-value and the number of genes enriched in this pathway. Among them, rich factor refers to the ratio of the number of genes located in this pathway among differentially expressed genes to the total number of genes located in this pathway among all annotated genes. The greater the rich factor, the greater the degree of enrichment. Q-value is the p-value after multiple hypothesis testing and correction. The range of $\mathrm{Q}$-value is $[0,1]$, which is closer to 0 , the more significant. We selected the most enriched 20 pathways for display in Fig. 8, among which biosynthesis of secondary metabolites, carbon metabolism and mRNA surveillance pathway are the three pathways with the largest number of enriched genes.

\section{Discussion}

Previous studies believed that circRNA was a byproduct of the transcription process (Cocquerelle et al. 1993; Memczak et al. 2013), but more and more studies in the past decade have confirmed that circRNA is a very important type of non-coding RNA, which plays a crucial role in a variety of biological processes (Chen et al. 2017a). Most of the researches on the function of circRNA have focused on mammals (Salzman et al. 2012), however, circRNA has also been identified in some plants, including Arabidopsis (Chen et al. 2017a), rice (Lu et al. 2015), wheat (Wang et al. 2017), and soybean (Wang et al. 2020). In present study, transcriptome sequencing and identification of the circRNA in the leaves of $B$. distachyon under dehydrated stress were performed. A total of 737 circRNAs were obtained, of which 
Table 2: Function classification of the predicted mRNA*

\begin{tabular}{|c|c|c|c|c|}
\hline \multirow{10}{*}{$\begin{array}{l}\text { Hormone } \\
\text { related }\end{array}$} & circRNA ID & Binding site of & target gene ID & Function annotation \\
\hline & $\begin{array}{l}\text { bdi_circ_0000045; bdi_circ_0000206; bdi_circ_0000214; } \\
\text { bdi_circ_0000257; bdi_circ_0000471; bdi_circ_0000472; } \\
\text { bdi_circ_0000483; bdi_circ_0000484; bdi_circ_0000485; } \\
\text { bdi_circ_0000489; bdi_circ_0000559; bdi_circ_0000576; } \\
\text { bdi_circ_0000581; bdi_circ_0000582; bdi_circ_0000629; } \\
\text { bdi_circ_0000708 } \\
\text { bdi_circ_0000045; bdi_circ_0000047; bdi_circ_0000206; } \\
\text { bdi_circ_0000214; bdi_circ_0000257; bdi_circ_0000366; } \\
\text { bdi_circ_0000471; bdi_circ_0000472; bdi_circ_0000483; } \\
\text { bdi_circ_0000484; bdi_circ_0000485; bdi_circ_0000517; } \\
\text { bdi_circ_0000559; bdi_circ_0000576; bdi_circ_0000581; } \\
\text { bdi_circ_0000582; bdi_circ_0000629; bdi_circ_0000708 }\end{array}$ & bdi-miR5174d-5p & Bradi3g40830.1 & auxin-responsive family protein \\
\hline & bdi_circ_0000130; bdi_circ_0000131; bdi_circ_0000214; & bdi-miR5174a & & \\
\hline & $\begin{array}{l}\text { bdi_circ_0000366; bdi_circ_0000471; bdi_circ_0000472; } \\
\text { bdi_circ_0000576; bdi_circ_0000581; bdi_circ_0000582 }\end{array}$ & bdi-miR5174b-5p & & \\
\hline & $\begin{array}{l}\text { bdi_circ_0000214; bdi_circ_0000471; bdi_circ_0000472; } \\
\text { bdi_circ_0000576; bdi_circ_0000581; bdi_circ_0000582 }\end{array}$ & $\begin{array}{l}\text { bd1-miks } 14 \mathrm{e}- \\
\text { bdi-miR5174c-5p }\end{array}$ & & \\
\hline & $\begin{array}{l}\text { bdi_circ_0000045 } \\
\text { bdi_circ_0000144 }\end{array}$ & $\begin{array}{l}\text { bdi-miR7757- } \\
\text { bdi-miR7708a-3p }\end{array}$ & $\begin{array}{l}\text { Bradilg46060.1 } \\
\text { Bradi1g46060.2 }\end{array}$ & $\begin{array}{l}\text { abscisic acid responsive } \\
\text { elements-binding factor } 2\end{array}$ \\
\hline & $\begin{array}{l}\text { bdi_circ_0000061 } \\
\text { bdi_circ_0000491; bdi_circ_0000492 }\end{array}$ & $\begin{array}{l}\text { bdi-miR171c-5p } \\
\text { bdi-miR5166 }\end{array}$ & $\begin{array}{l}\text { Bradi3g45880.9; Bradi3g45880.5; } \\
\text { Bradi3g04920.20 }\end{array}$ & auxin response factor \\
\hline & $\begin{array}{l}\text { bdi circ } 0000324 \text {; bdi circ } 0000325 \\
\text { bdi_circ_0000015 }\end{array}$ & $\begin{array}{l}\text { bdi-miR9486b } \\
\text { bdi-miR9491 }\end{array}$ & Bradi2g44490.2; Bradi2g44490.1 & auxin-like 1 protein \\
\hline & bdi_circ_0000082 & bdi-miR164f & Bradilg22830.1 & Auxin-responsive GH3 family \\
\hline & bdi_circ_0000330; bdi_circ_0000331 & bdi-miR528-3p & Bradi2g46190.3 & $\begin{array}{l}\text { Transcriptional factor B3 family } \\
\text { protein / auxin-responsive factor } \\
\text { AUX/IAA-related }\end{array}$ \\
\hline \multirow[t]{5}{*}{ Photosystem } & bdi_circ_0000491; bdi_circ_0000492 & bdi-miR5166 & $\begin{array}{l}\text { Bradilg24870.6; Bradilg24870.2; } \\
\text { Bradilg24870.5; Bradilg24870.4; } \\
\text { Bradilg24870.3; Bradilg24870.1 }\end{array}$ & $\begin{array}{l}\text { light harvesting complex } \\
\text { photosystem II }\end{array}$ \\
\hline & bdi_circ_0000366 & bdi-miR9495 & Bradilg77340.1; Bradilg77340.3 & $\begin{array}{l}\text { Mog1/PsbP/DUF1795-like } \\
\text { photosystem II reaction center } \\
\text { PsbP family protein }\end{array}$ \\
\hline & bdi_circ_0000041; bdi_circ_0000072; bdi_circ_0000395; & bdi-miR5169a & Bradi4g19720.1 & photosystem II reaction center \\
\hline & & bdi-miR5169b & Bradi4g19720.1 & protein $\mathrm{A}$ \\
\hline & $\begin{array}{l}\text { bdi_circ_0000483; bdi_circ_0000484; bdi_circ_0000485; } \\
\text { bdi_circ_0000632; bdi_circ_0000708 }\end{array}$ & bdi-miR159a-5p & Bradi1g25430.4 & \\
\hline \multirow[t]{5}{*}{ Stress response } & bdi_circ_0000294 & bdi-miR5064 & Bradilg28090.2 & $\begin{array}{l}\text { chloroplastic drought-induced } \\
\text { stress protein of } 32 \mathrm{kD}\end{array}$ \\
\hline & CircRNA ID & $\begin{array}{l}\text { Binding site of } \\
\text { mRNA }\end{array}$ & Target gene ID & Function annotation \\
\hline & bdi_circ_0000009 & $\begin{array}{l}\text { bdi-miR5281a } \\
\text { bdi-miR5281b }\end{array}$ & Bradi2g28040.2 & $\begin{array}{l}\text { Drought-responsive family } \\
\text { protein }\end{array}$ \\
\hline & $\begin{array}{l}\text { bdi_circ_0000041; bdi_circ_0000092 } \\
\text { bdi_circ_0000092 }\end{array}$ & bdi-miR7726a-5p & $\begin{array}{l}\text { Bradi3g34540.1; Bradi3g34540.5 } \\
\text { Bradi3g34540.4; Bradi3g34540.3 }\end{array}$ & $\begin{array}{l}\text { early-responsive to dehydration } \\
\text { stress protein (ERD4) }\end{array}$ \\
\hline & bdi_circ_0000308 & bdi-miR827-5p & $\begin{array}{l}\text { Bradi3g34540.6; Bradi3g34540.3; } \\
\text { Bradi3g34540.4; Bradi3g34540.5; } \\
\text { Bradi3g34540.1 }\end{array}$ & \\
\hline \multirow[t]{5}{*}{ Ion transport } & bdi_circ_0000617 & bdi-miR9496 & $\begin{array}{l}\text { Bradilg45940.7; Bradilg45940.8; } \\
\text { Bradilg45940.2 }\end{array}$ & high-affinity $\mathrm{K}^{+}$transporter 1 \\
\hline & bdi_circ_0000072 & bdi-miR437 & Bradi5g26820.1; Bradi4g01430.1 & $\mathrm{K}^{+}$efflux antiporter 1 \\
\hline & $\begin{array}{l}\text { bdi_circ_0000009 } \\
\text { bdi circ } 0000617\end{array}$ & $\begin{array}{l}\text { bdi-miR5281a } \\
\text { bdi-miR5281b } \\
\text { bdi-miR9496 }\end{array}$ & Bradi4g01430.1 & $\mathrm{K}^{+}$efflux antiporter 3 \\
\hline & bdi_circ_0000491; bdi_circ_0000492 & bdi-miR5166 & $\begin{array}{l}\text { Bradilg37860.9; Bradilg37860.5; } \\
\text { Bradilg37860.6; Bradilg37860.7; } \\
\text { Bradilg37860.8; Bradilg37860.11; } \\
\text { Bradilg37860.10; Bradilg37860.1; } \\
\text { Bradilg37860.12 }\end{array}$ & $\mathrm{K}^{+}$efflux antiporter 4 \\
\hline & bdi_circ_0000708 & bdi-miR169d & Bradilg76640.5 & $\mathrm{K}^{+}$efflux antiporter 5 \\
\hline \multirow[t]{4}{*}{$\begin{array}{l}\text { Regulation } \\
\text { factor }\end{array}$} & bdi_circ_0000461 & bdi-miR396e-5p & $\begin{array}{l}\text { Bradilg09900.2; Bradi3g52547.1; } \\
\text { Bradi5g20607.1; Bradi1g50597.1; } \\
\text { Bradi3g57267.1; Bradi3g39620.3; } \\
\text { Bradi3g39630.1; Bradi3g39590.1 }\end{array}$ & growth-regulating factor \\
\hline & bdi_circ_0000061 & bdi-miR171c-5p & Bradi2g23250.1 & heat shock protein 70 \\
\hline & bdi_circ_0000254 & bdi-miR5184 & $\begin{array}{l}\text { Bradi5g24410.6; Bradi5g24410.3; } \\
\text { Bradi5g24410.5; Bradi5g24410.1; } \\
\text { Bradi5g24410.2 }\end{array}$ & jasmonate-zim-domain protein 12 \\
\hline & bdi_circ_0000444 & bdi-miR5178-3p & $\begin{array}{l}\text { Bradi5g07080.3; Bradi5g07080.1; } \\
\text { Bradi5g07080.4; Bradi5g07080.2 }\end{array}$ & $\begin{array}{l}\text { Oxidoreductase, zinc-binding } \\
\text { dehydrogenase family protein }\end{array}$ \\
\hline
\end{tabular}

Table 2: Continued 
Table 2: Continued

\begin{tabular}{|c|c|c|c|c|}
\hline & CircRNA ID & $\begin{array}{l}\text { Binding site of } \\
\text { mRNA }\end{array}$ & target gene ID & Function annotation \\
\hline & bdi_circ_0000444 & bdi-miR5178-3p & $\begin{array}{l}\text { Bradi5g07080.3; Bradi5g07080.1; } \\
\text { Bradi5g07080.4; Bradi5g07080.2 }\end{array}$ & $\begin{array}{l}\text { Oxidoreductase, zinc-binding } \\
\text { dehydrogenase family protein }\end{array}$ \\
\hline & bdi_circ_0000461 & bdi-miR7738-5p & $\begin{array}{l}\text { Bradi2g58130.5; Bradi2g58130.4; } \\
\text { Bradi2g58130.6; Bradi2g58130.3; } \\
\text { Bradi2g58130.7 }\end{array}$ & relative of early flowering 6 \\
\hline & bdi_circ_0000228 & bdi-miR7774-5p & Bradilg59560.4 & splicing factor, putative \\
\hline & bdi_circ_0000276 & bdi-miR2118b & Bradi5g22310.7; Bradi5g22310.10; & starch synthase 3 \\
\hline & bdi_circ_0000367; bdi_circ_0000369; bdi_circ_0000370 & $\begin{array}{l}\text { bdi-miR7777- } \\
\text { 3p.1 }\end{array}$ & $\begin{array}{l}\text { Bradi5g22310.7; Bradi5g22310.10; } \\
\text { Bradi5g22310.5; Bradi5g22310.8; } \\
\text { Bradi5g22310.4; Bradi5g22310.6; } \\
\text { Bradi5g22310.3; Bradi5g22310.9; } \\
\text { Bradi5g22310.1; Bradi5g22310.2 }\end{array}$ & \\
\hline \multirow[t]{17}{*}{ TF } & bdi_circ_0000629 & bdi-miR7782-3p & $\begin{array}{l}\text { Bradi3g08280.2; Bradi3g08280.1; } \\
\text { Bradi3g08280.5; Bradi3g08280.3; } \\
\text { Bradi3g08280.4 }\end{array}$ & $\begin{array}{l}\text { basic helix-loop-helix (bHLH) } \\
\text { DNA-binding family protein }\end{array}$ \\
\hline & $\begin{array}{l}\text { bdi_circ_0000214; bdi_circ_0000471; bdi_circ_0000472; } \\
\text { bdi_circ_0000576; bdi_circ_0000581; bdi_circ_0000582 }\end{array}$ & bdi-miR5174d-3p & Bradi3g39927.1 & \\
\hline & $\begin{array}{l}\text { bdi_circ_0000617 } \\
\text { bdi_circ_0000366; bdi_circ_0000456; bdi_circ_0000489; } \\
\text { bdi_circ_0000490; bdi_circ_0000491; bdi_circ_0000511; } \\
\text { bdi_circ_0000531; bdi_circ_0000540 }\end{array}$ & $\begin{array}{l}\text { bdi-miR9496 } \\
\text { bdi-miR9493 }\end{array}$ & Bradilg54111.5 & \\
\hline & $\begin{array}{l}\text { bdi_circ_0000072; bdi_circ_0000130; bdi_circ_0000131; } \\
\text { bdi_circ_0000242; bdi_circ_0000439; bdi_circ_0000511; } \\
\text { bdi_circ_0000520; bdi_circ_0000521; bdi_circ_0000607; } \\
\text { bdi_circ_0000622; bdi_circ_0000623; bdi_circ_0000624; } \\
\text { bdi_circ_0000659; bdi_circ_0000660; bdi_circ_0000664; } \\
\text { bdi_circ_0000665; bdi_circ_0000682; bdi_circ_0000683; } \\
\text { bdi_circ_0000733; bdi_circ_0000734; bdi_circ_0000735 }\end{array}$ & $\begin{array}{l}\text { bdi-miR5171a } \\
\text { bdi-miR5171b }\end{array}$ & Bradilg05480.1 & $\begin{array}{l}\text { bZIP transcription factor family } \\
\text { protein }\end{array}$ \\
\hline & bdi_circ_0000045 & bdi-miR7757- & Bradi5g23340.3 & \\
\hline & bdi_circ_0000092 & bdi-miR7743-3p & $\begin{array}{l}\text { Bradilg46230.11; Bradilg46230.8; } \\
\text { Bradilg46230.13; Bradi1g46230.14; } \\
\text { Bradilg46230.12; Bradilg46230.9 }\end{array}$ & $\begin{array}{l}\text { global transcription factor group } \\
\text { A2 }\end{array}$ \\
\hline & bdi_circ_0000041; bdi_circ_0000092 & bdi-miR7726a-5p & Bradi3g05260.1 & K-box region and MADS-box \\
\hline & $\begin{array}{l}\text { bdi_circ_0000708 } \\
\text { bdi_circ_0000330; bdi_circ_0000331; bdi_circ_0000332; } \\
\text { bdi_circ_0000333; bdi_circ_0000334; bdi_circ_0000335 }\end{array}$ & $\begin{array}{l}\text { bdi-miR169d } \\
\text { bdi-miR5198 }\end{array}$ & Bradi3g54720.4 & $\begin{array}{l}\text { Plant-specific GATA-type zinc } \\
\text { finger transcription factor family } \\
\text { protein }\end{array}$ \\
\hline & bdi_circ_0000396 & bdi-miR5163a-3p & Bradilg08106.2 & WRKY DNA-binding protein \\
\hline & CircRNA ID & $\begin{array}{l}\text { Binding site of } \\
\text { mRNA }\end{array}$ & Target gene ID & Function annotation \\
\hline & $\begin{array}{l}\text { bdi_circ_0000013; bdi_circ_0000041; bdi_circ_0000088; } \\
\text { bdi_circ_0000144; bdi_circ_0000162; bdi_circ_0000164; } \\
\text { bdi_circ_0000165; bdi_circ_0000166; bdi_circ_0000167; } \\
\text { bdi_circ_0000211; bdi_circ_0000228; bdi_circ_0000334; } \\
\text { bdi_circ_0000335; bdi_circ_0000344; bdi_circ_0000366; } \\
\text { bdi_circ_0000458; bdi_circ_0000581; bdi_circ_0000582; } \\
\text { bdi_circ_0000687; bdi_circ_0000721; bdi_circ_0000736 }\end{array}$ & bdi-miR5175b & Bradilg08106.4 & \\
\hline & bdi_circ_0000045 & bdi-miR9494 & Bradi2g19070.1 & \\
\hline & bdi_circ_0000061 & bdi-miR171c-5p & Bradi4g33370.2; Bradi4g33370.9 & \\
\hline & bdi_circ_0000330; bdi_circ_0000331; bdi_circ_0000672 & bdi-miR528-5p & Bradi4g28280.1 & \\
\hline & bdi_circ_0000045 & bdi-miR9494 & Bradi2g42023.1 & \\
\hline & bdi_circ_0000288; bdi_circ_0000366 & bdi-miR164c-3p & Bradi2g30695.1 & \\
\hline & bdi_circ_0000172 & bdi-miR7771-3p & Bradi2g30800.2 & \\
\hline \multirow[t]{3}{*}{$\begin{array}{l}\text { Ribosomal } \\
\text { protein }\end{array}$} & bdi_circ_0000172 & bdi-miR7771-3p & Bradi3g09780.1 & $\begin{array}{l}\text { Late embryogenesis abundant } \\
\text { (LEA) hydroxyproline-rich } \\
\text { glycoprotein family }\end{array}$ \\
\hline & bdi_circ_0000172 & bdi-miR7771-3p & Bradi4g34750.1 & $\begin{array}{l}\text { Ribosomal protein } \\
\text { L7Ae/L30e/S12e/Gadd45 family } \\
\text { protein }\end{array}$ \\
\hline & bdi_circ_0000045 & bdi-miR7757- & Bradi1g71200.1 & Ribosomal protein S3Ae \\
\hline
\end{tabular}

* Part of the classification results is displayed. See Supplementary Table 2 for the complete results

exon circRNA exceeded $50 \%$ (Fig. 3). The high ratio of exon circRNA is similar with the Arabidopsis exon circRNA under high temperature (Pan et al. 2018). It is speculated that the increase in the number of exon circRNA may be due to adversity stress. The increase in exon circRNA not only responds to plant stress, but may also enhance stress resistance. It has been confirmed that circRNA in plants can respond to a variety of stresses. Zuo et al. (2016) verified that 163 circRNAs in tomato respond to cold stress, while 62 circRNAs in wheat are differentially expressed under drought stress compared to the control (Wang et al. 2017). There are 597 circRNAs with significant differential 
circRNAs Respond to Drought in B. distachyon / Intl J Agric Biol, Vol 25, No 6, 2021

Table 3: Statistics of circRNA expression in seven functional categories

\begin{tabular}{lccc}
\hline Function classification & circRNA & Up-regulated circRNA & Down-regulated circRNA \\
\hline Hormone related & 124 & 78 & 46 \\
Photosystem & 47 & 27 & 10 \\
Stress response & 30 & 20 & 13 \\
Ion transport & 34 & 21 & 38 \\
Regulation factor & 94 & 56 & 53 \\
TF & 141 & 88 & 51 \\
Ribosomal protein & 130 & 79 & \\
\hline
\end{tabular}

expression in the leaves of $B$. distachyon under dehydration stress, which is consistent with previous research results, that is, circRNA can respond to multiple stresses and may further participate in the regulation of stress resistance.

The predicted target genes of circRNAs were analyzed by bioinformatics. The GO results showed that they were related to multiple functions, including different biological processes, cellular components, and molecular functions. EGG analysis enriched 20 pathways, most of which were involved in drought response. For example, the pathway with the largest number of enriched genes is biosynthesis of secondary metabolites, and there are as many as 89 hormone-related genes in the functional classification of target genes, including auxin-responsive family protein, auxin-responsive factor, and abscisic acid responsive elements-binding factor. Phytohormones are active substances induced by plant cells receiving specific environmental signals. They not only regulate plant growth and development, but also participate in a variety of stress responses, including low temperature (Xin et al. 2019), water deficit (Hu et al. 2018), salt (Yu et al. 2020) and so on (Wang et al. 2018). The exogenous application of ABA can increase the cold tolerance of rice plants during the flowering period and thus increase the seed setting rate (Xiang et al. 2019). Besides, exogenous application of gibberellin could relieve the adverse effects of salinity, drought, and heat stresses on the growth of Capsicum аппиит L., including increase biomass and chlorophyll content, as well as improve photosynthetic efficiency (Khan et al. 2015). Since circRNA can act as a molecular sponge of miRNA and prevent it from regulating target mRNA and controlling gene expression (Memczak et al. 2013), the expression trend of most circRNAs is positively correlated with their corresponding target genes. Under drought treatment, there were more markedly up-regulated circRNA in the leaves of $B$. distachyon than down-regulated expression. For example, the circRNAs predicted to be hormone-related genes, 78 were up-regulated and 46 were down-regulated. Therefore, it is speculated that the circRNA corresponding to the hormone-related gene is involved in dehydrated response of $B$. distachyon in a positive or negative manner, and contributes to the regulation of its stress response.

In addition to hormone-related genes, the circRNA corresponding to target genes whose function is annotated as stress response, regulation factor, photosystem and ion transport are also up-regulated more than down-regulated. Drought is one of the common environmental stresses. Genes that respond to drought stress have always been research hotspots. With the continuous development of high-throughput sequencing technology, the function of non-coding RNA is gradually recognized by people. Apart from miRNA and IncRNA, circRNA in plants also play a significant role in stress resistance. The circRNA and its target genes obtained in this analysis can be divided into seven categories: hormone-related, photosystem, stress response, regulation factor, ion transport, TF and ribosomal protein according to their functional classification. Among them, photosystem- and hormone-pathway, as known regulation networks, participate in plant drought response (Chen et al. 2016; Rao and Chaitanya 2016), including a series of responses, like light harvesting complex photosystem II, photosystem II reaction center protein A, auxin response factor and auxin transport protein genes, are involved in dehydration response, which is consistent with previous studies (Chen et al. 2016; Rao and Chaitanya 2016). Furthermore, Rai et al. (2012) confirmed that earlyresponsive to dehydration stress protein (ERD4) is highly expressed in plant drought response and could effectively improve the desiccation adaptability of plants. ERD4 in the stress response classification was up-regulated under drought treatment, which was in line with the results of Rai et al. Under adversity stress, a large number of genes are upregulated and expressed to positively regulate the stress resistance of plants, whereas these emergency response genes are inhibited by related mechanisms in normal growth conditions. During the evolution process, plants have established related mechanisms to inhibit the expression of stress-related genes under normal conditions. The main role of growth-regulating factors (GRFs) is to inhibit the stress response under non-stress conditions. Compared with wildtype Arabidopsis and AtGRF7 overexpression lines, the Arabidopsis atgrf7 mutant is more tolerant to salt and dehydrated stress (Kim et al. 2012), therefore, GRF is down-regulated under stress conditions to activate related stress genes to improve plant resistance. The circRNAs, whose target genes belong to the growth-regulating factor classification, were distinctly down-regulated under drought treatment, which is consistent with the negative regulation of plant stress resistance by GRF, which may be a crucial regulatory network of stress response in the plant.

The $\mathrm{K}^{+}$efflux antiporter (KEA), for $\mathrm{K}^{+}$, is functioning 
in maintaining $\mathrm{pH}$, the active accumulation and balance of $\mathrm{K}^{+}$and ion homeostasis (Zhu et al. 2018). Additionally, soybean GmKEAs gene family up-regulated in the beginning and down-regulated later under excessive potassium stress (Tao et al. 2015). The response of GmKEAs gene to abiotic stress indicates that it is involved in soybean resistance. The target genes, whose function annotated as KEA1, KEA3, KEA4 and KEA5 in B. distachyon, their corresponding circRNAs were also up-regulated after water shortage, which may also be involved in the response to osmotic stress. WRKY TF exerts a critical role in a variety of biological processes, including plant growth, development and adversity stress. Gao et al. (2018) overexpressed TaWRKY2 in wheat to enhance the dehydrated resistance of transgenic plants. Shirazi et al. (2019) cloned the stress response BnWRKY57 in Brassica napus and analyzed its expression. Those results showed that BnWRKY57 not only responded to drought and salt stress, but also enhanced the resistance of Brassica napus to the two stresses. The circRNA whose target gene function was annotated as WRKY DNA-binding protein was obviously up-regulated after drought treatment, and it was speculated that it not only participated in the drought response of $B$. distachyon, but also exerted an influence in stress defense.

Moreover, some of the other predicted target genes in the functional classification may also be involved in the drought response of $B$. distachyon. However, more evidence is needed to further prove its specific function, and the mechanism of action may depend on circRNA regulation.

\section{Conclusion}

Through transcriptome sequencing of $B$. distachyon leaves under drought treatment, a total of 737 circRNAs were obtained in this study, of which exon circRNA exceeded $50 \%$. Differential expression analysis showed that there were 332 and 265 up-regulated and down-regulated expressions, respectively and 140 had no significant difference. There were 229 and 303 circRNAs specifically expressed under CK and drought, respectively, and 204 were co-expressed. Bioinformatics methods were used to identify potential miRNA targets of differentially expressed circRNAs. Among them, 150 differentially expressed circRNAs had putative miRNA binding sites. Based on the predicted miRNA with binding site, the target gene downstream of the miRNA was screened. Analysis of GO and KEGG shows that the categories of metabolic process (GO: 0008152), cell (GO: 0005623) and cell part (GO: 0044464), binding (GO: 0005488) and catalytic activity (GO: 0003824) are the most abundant entry in GO. Among the 20 most significant pathway entries, biosynthesis of secondary metabolites, carbon metabolism and mRNA surveillance pathway are the three pathways with the largest number of enriched genes. According to the functional annotation of target genes, they are divided into seven categories: hormone-related, photosystem, stress response, regulation factor, ion transport, TF and ribosomal protein. CircRNA is an important type of non-coding RNA in organisms, and these circRNAs involved in desiccation response may exert a crucial role in drought response and defense of $B$. distachyon. The valuable information also provides a theoretical basis for further identification of candidate genes participated in drought regulation.

\section{Acknowledgements}

This research was financially supported by the Natural Science Foundation of Henan Province (Grant No. 202300410133), the Key Research Projects of Higher Education in Henan Province (Grant No. 19B210002), and Student Research Training Program of Henan University of Science and Technology (Grant No. 2020338 and 2020335).

\section{Author Contributions}

Yalan Feng performed the experiments and wrote the manuscript; Shuang Zhou and Jun Zhang analyzed the experiment data and wrote the manuscript; Ke Xv, Yating Li and Mengzhen Zhang sampled material and analyzed the experiment data; Chao Ma and Youjun Li designed the research and revised the manuscript.

\section{Conflict of Interest}

Authors declare no conflict of interest.

\section{Data Availability}

The data and supplementary material are all available online.

\section{Ethics Approval}

This research does not involve the ethical approval.

\section{References}

Behrooz D, N Shahin, B Søren (2016). Identification of circular RNAs from the parental genes involved in multiple aspects of cellular metabolism in barley. Front Plant Sci 7; Article 776

Brkljacic J, E Grotewold, R Scholl, T Mockler, DF Garvin, P Vain, T Brutnell, R Sibout, M Bevan, H Budak, AL Caicedo, C Gao, Y Gu, SP Hazen, BF Holt, SY Hong, M Jordan, AJ Manzaneda, T MitchellOlds, K Mochida, LAJ Mur, CM Park, J Sedbrook, M Watt, SJ Zheng, JP Vogel (2011). Brachypodium as a model for the grasses: today and the future. Plant Physiol 157:3-13

Catalan P, D López-Álvarez, A Díaz-Pérez, R Sancho, ML López-Herránz (2015). Phylogeny and evolution of the genus Brachypodium. In: Genetics and genomics of Brachypodium, pp:9-38. Springer, Cham, Switzerland

Chen G, J Cui, L Wang, Y Zhu, Z Lu, B Jin (2017a). Genome-wide identification of circular RNAs in Arabidopsis thaliana. Front Plant Sci 8; Article 1678

Chen L, YY Yu, XCZhang, C Liu, CY Ye, LJ Fan (2016). PcircRNA_finder: a software forcircRNA prediction in plants. Bioinformatics 32:3528-3529 
Chen QS, M Li, ZC Zhang, WW Tie, X Chen, LF Jin, N Zhai, QX Zheng, JF Zhang, R Wang (2017b). Integrated mRNA and microRNA analysis identifies genes and small miRNA molecules associated with transcriptional and post-transcriptional-level responses to both drought stress and re-watering treatment in tobacco. BMC Genomics 18; Article 62

Cocquerelle C, B Mascrez, D Hétuin, B Bailleul (1993). Mis-splicing yields circular RNA molecules. FASEB J 7:155-160

Dai XB, XP Zhao (2011). psRNATarget: A plant small RNA target analysis server. Nucl Acids Res 39:155-159

Fan X, X Zhang, X Wu, H Guo, Y Hu, F Tang, Y Huang (2015). Single-cell RNA-seq transcriptome analysis of linear and circular RNAs in mouse preimplantation embryos. Genome Biol 16:148-164

Feng YL, F Yin, YY Zhao, K Xv, J Zhang, Y Xiong, C Ma (2020). Transcriptional analysis of IncRNAs in Brachypodium distachyon under drought stress. Intl J Agric Biol 24:1-10

Feng YL, YY Zhao, C Ma, J Zhang, Y Xiong, J Yin (2019). Identification of photoperiod responsive genes in wheat cultivar Jing 841 by transcriptome sequencing. Intl J Agric Biol 22:1093-1110

Gao H, Y Wang, P Xu, Z Zhang (2018). Overexpression of a WRKY transcription factor TaWRKY2 enhances drought stress tolerance in transgenic wheat. Front Plant Sci 9; Article 997

Hansen TB, TI Jensen, BH Clausen, JB Bramsen, B Finsen, CK Damgaard, JR Kjems (2013). Natural RNA circles function as efficient microRNA sponges. Nature 495:384-388

Hu L, Y Xie, SJ Fan, ZS Wang, LG Kong (2018). Comparative analysis of root transcriptome profiles between drought-tolerant and susceptible wheat genotypes in response to water stress. Plant Sci 272:276-293

Iqbal M, S Ul-Allah, M Naeem, M Ijaz, A Sattar, A Sher (2017). Response of cotton genotypes to water and heat stress: From field to genes. Euphytica 213; Article 131

Jeck WR, JA Sorrentino, K Wang, MK Slevin, CE Burd, J Liu, WF Marzluff, NE Sharpless (2013). Circular RNAs are abundant, conserved, and associated with ALU repeats. RNA 19:141-157

Kanehisa M, M Araki, S Goto, M Hattori, M Hirakawa, M Itoh, T Katayama, S Kawashima, S Okuda, T Tokimatsu, Y Yamanishi (2008). KEGG for linking genomes to life and the environment. $\mathrm{Nucl}$ Acids Res 36:480-484

Khan AL, M Waqas, IJ Lee (2015). Resilience of Penicillium resedanum LK6 and exogenous gibberellin in improving Capsicum annuиm growth under abiotic stresses. J Plant Res 128:259-268

Kim JS, J Mizoi, S Kidokoro, K Maruyama, J Nakajima, K Nakashima, N Mitsuda, Y Takiguchi, M Ohme-Takagi, Y Kondou (2012) Arabidopsis growth-regulating factor7 functions as a transcriptional repressor of abscisic acid- and osmotic stress-responsive genes, including DREB2A. Plant Cell 24:3393-3405

Kos A, R Dijkema, AC Arnberg, PH Van, H Schellekens (1986). The hepatitis delta $(\mid[$ delta] $])$ virus possesses a circular RNA. Nature 323:558-560

Lu TT, LL Cui, Y Zhou, CR Zhu, DL Fan, H Gong, Q Zhao, CC Zhou, Y Zhao, DF Lu, JH Luo, YC Wang, QL Tian, Q Feng, T Huang, B Han (2015). Transcriptome-wide investigation of circular RNAs in rice. RNA 21:2076-2087

Ma C, J Zhang, JL Yuan, JR Guo, Y Xiong, YL Feng (2019). Differential expression of microRNAs are responsive to drought stress and exogenous Methyl Jasmonate in wheat (Triticum aestivum). Intl $J$ Agric Biol 22:475-486

Memczak S, M Jens, A Elefsinioti, F Torti, J Krueger, A Rybak, L Maier, SD Mackowiak, LH Gregersen, M Munschauer, A Loewer, U Ziebold, M Landthaler, C Kocks, Fl Noble, N Rajewsky (2013). Circular RNAs are a large class of animal RNAs with regulatory potency. Nature 495:333-338

Nadarajah K, IS Kumar (2019). Drought response in rice: The miRNA story. Intl J Mol Sci 20:3766-3785

Pan T, XQ Sun, YX Liu, H Li, GB Deng, HH Lin, SH Wang (2018). Heat stress alters genome-wide profiles of circular RNAs in Arabidopsis Plant Mol Biol 96:217-229

Rai A, P Suprasanna, SF D'Souza, V Kumar (2012). Membrane topology and predicted RNA-binding function of the'early responsive to dehydration (ERD4)' plant protein. PLoS One 7; Article e32658
Rao DE, KV Chaitanya (2016). Photosynthesis and antioxidative defense mechanisms in deciphering drought stress tolerance of crop plants. Biol Plantarum 60:1-18

Salzman J, C Gawad, PL Wang, N Lacayo, PO Brown (2012). Circular RNAs are the predominant transcript isoform from hundreds of human genes in diverse cell types. PLoS One 7; Article e30733

Sanger HL, G Klotz, D Riesner, HJ Gross, AK Kleinschmidt (1976). Viroids are single-stranded covalently closed circular RNA molecules existing as highly base-paired rod-like structures. Proc Natl Acad Sci 73:3852-3856

Sattar A, A Sher, M Ijaz, S Ul-Allah, M Butt, M Irfan, MS Rizwan, H Ali, MA Cheema (2020a). Interactive effect of biochar and silicon on improving morpho-physiological and biochemical attributes of Maize by reducing drought hazards. J Soil Sci Plant Nutr 20:1819-1826

Sattar A, A Sher, M Ijaz, S Ul-Allah, MS Rizwan, M Hussain, K Jabran, MA Cheema (2020b). Terminal drought and heat stress alter physiological and biochemical attributes in flag leaf of bread wheat. PLoS One 15; Article e0232974

Scholthof KBG, S Irigoyen, P Catalan, KK Mandadi (2018). Brachypodium: a monocot grass model genus for plant biology. Plant Cell 30:1673-1694

Shen YD, XW Guo, WM Wang (2017). Identification and characterization of circular RNAs in zebrafish. FEBS Lett 591:213-220

Shirazi FA, H Razi, A Niazi, A Alemzadeh (2019). Molecular cloning and expression analysis of a stress-responsive WRKY transcription factor gene, BnWRKY57, from Brassica napus. Plant Omics 12:37-47

Tao CH, C Xin, WB Yue, YX Xing, ZH Mei, CX Yan, LX Qing (2015). Whole-genome identifcation and expression analysis of $\mathrm{K}^{+}$efflux antiporter (KEA) and $\mathrm{Na}^{+} / \mathrm{H}^{+}$antiporter (NHX) families under abiotic stress in soybean. J Integr Agric 14:1171-1183

Wang L, Z Feng, X Wang, X Wang, X Zhang (2010). DEGseq: An R package for identifying differentially expressed genes from RNA-seq data. Bioinformatics 26:136-138

Wang X, X Chang, Y Jing, J Zhao, Q Fang, M Sun, Y Zhang, W Li, Y Li (2020). Identifcation and functional prediction of soybean circRNAs involved in low-temperature responses. J Plant Physiol 250:153188

Wang XL, RR Qin, RH Sun, JJ Wang, XG Hou, L Qi, J Shi, XL Li, YF Zhang, PH Dong (2018). No post-drought compensatory growth of corns with root cutting based on cytokinin induced by roots. Agric Water Manage 205:9-20

Wang Y, M Yang, S Wei, F Qin, H Zhao, B Suo (2017). Identifcation of circular RNAs and their targets in leaves of Triticum aestivum L. under dehydration stress. Front Plant Sci 7; Article 2024

Westholm JO, P Miura, S Olson, S Shenker, B Joseph, P Sanfilippo, SE Celniker, BR Graveley, EC Lai (2014). Genome-wide analysis of drosophila circular RNAs reveals their structural and sequence properties and age-dependent neural accumulation. Cell Rep 9:1966-1980

Xiang HT, DQ Qi, W Li, DF Zheng, YX Wang, TT Wang, LZ Wang, XN Zeng, CJ Yang, H Zhou, HD Zhao (2019). Effect of exogenous ABA on the endogenous hormone levels and physiology of chilling resistance in the leaf sheath of rice at the flowering stage under low temperature stress. Acta Pratacult Sin 28:81-94

Xin H, NX Chao, X Pan, L Wei, Y Min, K Yu, QL Wen, H Wei (2019). Comparative transcriptome analyses revealed conserved and novel responses to cold and freezing stress in Brassica napus L. G3 Genes Genome Genet 9:2723-2737

Xu Y, Y Ren, T Lin, D Cui (2019). Identification and characterization of circRNAs involved in the regulation of wheat root length. Biol Res $52: 19-26$

Ye CY, X Zhang, Q Chu, C Liu, Y Yu, W Jiang, QH Zhu, L Fan, L Guo (2016). Full-length sequence assembly reveals circular RNAs with diverse non-GT/AG splicing signals in rice. RNA Biol 14: 1055-1063

Ye CY, L Chen, C Liu, QH Zhu, L Fan (2015). Widespread noncoding circular RNAs in plants. New Phytol 208:88-95

Yu ZP, XB Duan, L Luo, SJ Dai, ZJ Ding, GM Xia (2020). How plant hormones mediate salt stress responses. Trends Plant Sci 25:1117-1130

Zhang H, B Yang, J Liu, D Guo, J Hou, S Chen, B Song, C Xie (2017). Analysis of structural genes and key transcription factors related to anthocyanin biosynthesis in potato tubers. Sci Hortic 225:310-316 
Feng et al. / Intl J Agric Biol, Vol 25, No 6, 2021

Zhao W, S Chu, YQ Jiao (2019). Present scenario of circular RNAs (circRNAs) in plants. Front Plant Sci 10; Article 379

Zhou L, J Chen, Z Li, X Li, X Hu, Y Huang, X Zhao, C Liang, Y Wang, L Sun, M Shi, X Xu, F Shen, M Chen, Z Han, Z Peng, Q Zhai, J Chen, Z Zhang, R Yang, J Ye, Z Guan, H Yang, Y Gui, J Wang, Z Cai, X Zhang (2010). Integrated profiling of microRNAs and mRNAs: MicroRNAs located on Xq27.3 associate with clear cell renal cell carcinoma. PLoS One 5; Article e15224
Zhu XJ, T Pan, X Zhang, LG Fan, FJ Quintero, H Zhao, XM Su, XJ Li, I Villalta, I Mendoza, JB Shen, LW Jiang, JM Pardo, QS Qiu (2018). $\mathrm{K}^{+}$efflux antiporters 4 , 5, and 6 mediate $\mathrm{pH}$ and $\mathrm{K}^{+}$ homeostasis in endomembrane compartments. Plant Physiol 178:1657-1678

Zuo JH, Q Wang, BZ Zhu, YB Luo, LP Gao (2016). Deciphering the roles of circRNAs on chilling injury in tomato. Biochem Biophys Res Commun 479:132-138 